\title{
The other nanotech
}

\section{The commercial success of low-tech nano-enabled products, such as sports equipment and lubricants, could pave the way for more advanced nanodevices and applications.}

The different ways that science can be funded and managed have been the subject of much debate in Nature Nanotechnology over the past two years. Some have argued that nanoscience and technology are 'post-academic' (ref. 1): in other words, research in the field is largely funded (by governments) to solve specific problems of interest to industry, and often involves collaborations across disciplines and between universities and companies. This stands in contrast to the traditional academic model in which knowledge is pursued for its own sake, academics are free to do 'blue-skies' research, and any benefits to society accrue in a trickledown, but unpredictable, fashion. For some, university research should move back to this model ${ }^{2}$. However, as is often the case, reality blurs the line between the models, as highlighted in Patrick McCray's article about the history of one

technology - giant magnetoresistance on page 2 of this issue. Moreover, many of the arguments that are marshalled to support the traditional academic view can also be used to support the post-academic view, and vice versa ${ }^{3}$.

While this debate continues, an interesting intersection has occurred. According to Lux Research ${ }^{4}, 2007$ was the first year in which companies around the world spent more on nanotechnology research (approximately $\$ 6.6$ billion) than governments ( $\$ 6.2$ billion). This corporate research comes on the back of what Lux estimates to be $\$ 147$ billion of "nanoenabled products produced in 2007". And, as with many other industries, nanotech companies have started by going for the 'low-hanging fruit'.

The recent National Nano Engineering Conference $^{5}$ in Boston was full of examples. Nanoblox, Inc., for example, sells its nanodiamonds for applications in lubrication, polishing and abrasive coatings (among others). Nanocomp Technologies is weaving nanotubes into sheets that can be used as flame retardants on body armour. Luna nanoWorks has noticed that fullerenes seem to encourage hair regrowth in mice. Many more examples are included in the list of nanotechnology consumer products maintained by the Project on Emerging Nanotechnologies ${ }^{6}$. There, one finds glues, degreasers, baby bottles and stain-resistant trousers next to $16 \mathrm{~Gb}$ NAND flash. Famously, there are also the sports equipment applications - the Stealth nanotube baseball bat, the Accuflex Evolution Golf Shaft and the MAX NANO badminton racquet.

\section{Nanotech companies have started by going for the 'low-hanging fruit'.}

These applications - which we might call the 'other nanotech' - are distinctly more prosaic than the single-electron transistors, molecular switches and other advanced nanotechnology that are explored in many university laboratories. For the other nanotech, fundamental understanding can be less important than practical considerations: how can a technology be mass-produced? How can it be packaged? How stable is it? In addition, the technologies themselves are often based on basic material properties: the ability of nanoparticles to be strong, hard, small and conductive (of heat and electricity).

Is this other nanotech an example of post-academic science? Maybe. What is clear though is that the sheer breadth of fields encompassed by nanotechnology prevents a single scientific management model from being complete. Although the simultaneous existence of differing modes of nanoscience and technology research inevitably leads to competition with funding for blue-skies research often, and regrettably, under particular stress - it should prove advantageous in the long term. This is because expertise, equipment and revenue will be built on the backs of sunblocks, tennis racquets and other examples of the other nanotech, in advance of the arrival of more sophisticated, demanding and expensive work.

Let's return to Nanoblox. At the same time that their nanodiamonds are being mixed into lubricants for trucking fleets, the company is developing a purified version of the same material as a drug-delivery platform - one of the most challenging and potentially lucrative applications of nanotechnology. Just like Corning Glass, who found themselves in an ideal position to become a fibre-optics manufacturer when that technology arrived, a manufacturer of nanocreams might parlay its expertise into cancer treatment at the right moment.

Just as nanofabrication benefits from the co-existence of the top-down and bottomup approaches, the whole nanosector can benefit from the co-existence of advanced nanotech and the other nanotech, as well as from a variety of managerial and funding models.

\footnotetext{
References

1. Vogt, T., Baird, D. \& Robinson, C. Nature Nanotech. 2, 329-332 (2007).

2. Moriarty, P. Nature Nanotech. 3, 60-62 (2008).

3. Jones, R. Nature Nanotech. 3, 448-449 (2008).

4. <www.luxresearchinc.com/press/RELEASE Nano-SMR 7_22_08.pdf > (22 July 2008).

5. <www.nasatech.com/nano/index.html>

6. <www.nanotechproject.org/inventories>
} 